\title{
Effect of Sample Matrix on Radial and Axial Profiles of Ion Abundance in Inductively Coupled Plasma Mass Spectrometry
}

\author{
Clarisse Mariet ${ }^{1 *}$, Francine Carrot ${ }^{2}$, Mélanie Moskura ${ }^{1}$ \\ ${ }^{1}$ Laboratoire Interdisciplinaire sur l'Organisation Nanométrique et Supramoléculaire (LIONS), \\ CEA Saclay Gif-sur Yvette, France \\ ${ }^{2}$ UMR 3299, CEA Saclay Gif-sur Yvette, France \\ E-mail: ${ }^{*}$ clarisse.mariet@cea.fr \\ Received April 30, 2011; revised June 26, 2011; accepted July 8, 2011
}

\begin{abstract}
In inductively coupled plasma mass spectrometry (ICP-MS) analysis, only a few options are available to deal with non-spectroscopic interferences. Considering that diluting the sample is impractical for traces analysis, other alternatives must be employed. Traditionally, the method of standard additions is used to correct the matrix effect but it is a time consuming method. Others methods involves separation techniques. Another way to overcome matrix interferences is to understand the mechanism involved and adjust plasma viewing conditions to reduce or eliminate the effect. In this study, the effect of various concomitant elements in ICP-MS was assessed by measuring the distribution of selected singly charged analyte ions ( $\mathrm{Al}, \mathrm{V}, \mathrm{Cr}, \mathrm{Mn}$, $\mathrm{Ni}, \mathrm{Co}, \mathrm{Cu}, \mathrm{Zn}, \mathrm{As}$, In, Ba, La, Ce, Pb), doubly charged ions ( $\mathrm{La}, \mathrm{Ce}, \mathrm{Ba}$ and $\mathrm{Pb}$ ) and oxides ions (BaO) in the presence of concomitant elements spanning a mass range from 23 (Na) to 133 (Cs) u.m.a. and different ionization energies. Concomitant elements are alkali metals, alkaline earth metals and Si. Analyte ion suppression was observed while moving the ICP across and away from the sampling interface with or without a single concomitant element. Matrix effect measures were realised, firstly, to highlight the relation between the signal extinction of an analyte and the masse of the concomitant element, and secondly to highlight the relation between the removal of the analyte signal and the first ionization energy of the element of matrix. A dependence upon both the mass of the matrix element and the mass of the analyte was observed. The suppression seems increased with increasing matrix element mass and decreased with increasing analyte mass. The effect of the mass of the matrix element was the more significant of the two factors. If space-charge effects were found to be significant for matrix elements of much lower mass, it seems diffusion also played an active part for heavier matrix elements. Finally, some evidence was found for a shift in ion-atom equilibrium for dications and for energy demand regarding oxides.
\end{abstract}

Keywords: Matrix Effects, Easily Ionized Elements, Inductively Coupled Plasma Mass Spectrometry, Spatial Profiling

\section{Introduction}

Inductively coupled plasma mass spectrometry (ICP-MS) is a well-established analytical technique. Ever since the development of ICP-MS in the mid 1980s, the determination of trace levels of certain elements in high matrix samples can be considered a "classical" difficult application. As a result of the rather invasive sampling process, ICP-MS is much more susceptible to effects of concomitant elements (also called matrix effect (ME) and non -spectroscopic interferences) than inductively coupled plasma atomic emission spectrometry (ICP-AES). These interferences are usually observed as a suppression of analyte signals, although enhancements have also been report. The ME is probably one of the most crucial limitations leading to a possible deterioration of the accuracy of analysis. A considerable number of reports have been dedicated to the study of the ME since 1979 [1-4], notably with easily ionizable element (EIE) such as sodium or calcium. The matrix effect due to $\mathrm{Ca}$ is more severe 
than that due to $\mathrm{K}$ or $\mathrm{Na}$ and is well-known but the exact mechanism of the ME is still ignored [5]. Because the matrix effects of $\mathrm{Ca}$ and $\mathrm{Na}$ are of different origin, it is easy to eliminate the ME from Na by choosing operating conditions under which plasma is robust (good radial and axial view modes) whereas one can only slightly decrease the effects of matrix created by Ca [6]. The same applies with acids or solvents [7-9]. Because of their origin, only a few options are available to deal with non-spectroscopic interferences. Considering that reducing the amount of material loaded into the plasma (which can be done by diluting the sample) is impractical for traces analysis, since it would result in less analyte atoms measured, other alternatives must be employed [10]. Traditionally, in plasma spectrometry, the EIE effect is mitigated either by matrix matching the calibration solutions with the sample, something not always easily done [5]. It is also possible to use the method of standard additions $[11,12]$ but it is a time consuming method. Several methods have been proposed for alleviating these interferences and can be classified into two groups depending on whether separation techniques are involved or not. The first group involves separation techniques $[13,14]$. Second group applies physical techniques like electrothermal vaporization [15-16], which had limitations such as non-spectroscopic interference, which might occur when a high salt content sample was analyzed.

In our point of view, the best way to overcome matrix interferences is to understand the mechanism involved and adjust plasma viewing conditions to reduce or eliminate the effect. Studies relating to ICP-AES $[5,6]$ or ICP-MS propose several possible origins with the effects of matrix related to plasma. The most commonly cited origins are the ion-electron recombinations $[5,6,18,19]$, the energy demand $[5,6,18]$, increase in the collisions [6], the space charge effect [19] and change the spatial distribution of the species in the source with lateral $[5,6,18]$ or ambipolar diffusion $[5,6,18,19]$.

So as to bring out the parameters influencing ME, we suggest describing each of the non-spectroscopic interferences origins listed here. A displacement of the ionization equilibrium is the simplest explanation of the signal extinction. Although the presence of EIE does not modify significantly the total number of electrons in the plasma, it causes an important increase of the charge density along the axis of the plasma [19] and a temperature decrease. The higher charge density moves the equilibrium towards the atomic form. The modification of the ion-atom equilibrium for an analyte translates by the maximum intensity of the signal along the central axis of the plasma. Ions are formed in zones of higher temperatures, closer to the sampler. There is a delay in the ionization. To observe the maximum intensity of the signal, it is then necessary to increase the distance torch-sampler.

According to thermodynamic calculations, there should not be refractory oxides in plasma. However, as the drops size is not always identical, oxides are formed. Oxides exist mainly in the coldest zones of plasma, closer to the torch and on the edges of the flame. For an analyte $\mathrm{M}$, signals of the ions $\mathrm{MO}^{+}$and $\mathrm{MOH}^{+}$are observed while moving away plasma from the sampler. If the formation of oxide is related to a reduction in the temperature, the maximum of intensity of the $\mathrm{MO}^{+}$signal will be observed at a longer distance from the sampler. If, indeed, the formation of oxide is related to a problem of atomizing, the peak of maximum intensity does not vary with the position of observation. Thus the formation of oxides on the edges of plasma can be highlighted by an abrupt modification of the signal when the position of observation is changed.

The addition of a concomitant element modifies the energy demand in plasma according to the concentration and the energy of ionization of this element.

The first ones to have mentioned the space charge effect are Olivares and Houk [20]. This ME involves the positively charged lenses that collect the positive ions, at the exit of the skimmer, by confining them in a beam. According to them, the strong ionic currents in ICP exceed the maximum current allowing neglecting the charge effect. The ionic current is inversely proportional to the ion mass [19]. However, the ions beam is delimited by the total current in the beam and repulsion forces. Consequently, there is a broadening of the beam especially since the ions are light and, simultaneously, transmission of the light ions is less effective (part of them is lost in way) than that of heavy ones. Lighter ions, with stronger density of charge and weaker kinetic energy than heavy ions, are more easily deviated. It is what explains the greatest sensitivity for the heavy ions that for the light ones $(1 \mathrm{~Pb}$ corresponds to approximately 120.000 counts for $\mathrm{U}$ and 3000 counts for Li). Thus a matrix of heavy elements will extinguish the signal more strongly than a matrix of light elements. The signal widens with the addition of a heavy matrix element whatever is the observation position in plasma since the increase in the diffusion does not take place in plasma but behind the cones, in the interface. Chen and Houk [21] observed it is possible to attenuate the extinction created per 1000 Cs ppm on 50 Sc ppm with a weak loss of sensitivity by modifying the potential of the lenses.

In the same way that the ionization of an analyte can be delayed by the addition of an element of matrix, the vaporization of the element can take place in zones of plasma closer to the sampler. The signal thus decreases, for the same position of observation and widens because 
the delay of vaporization allows a greater diffusion. One speaks about lateral diffusion. Spatial change distribution can take place in another way. In plasma, the electrons and the ions diffuse towards the areas of lower charge density. By consequent the ions and the electrons move away from the source of arrival of the sample. The electrons diffuse more quickly than the ions. Consequently, locally there are zones where neutrality is not respected; forces of repulsion appear to restore it. Because of the created electric field the electrons are slow whereas they are accelerated by the repelling powers. The sum of these two phenomena leads to a total diffusion and the same speed of the ions and electrons. The larger charge density is, the more the diffusion increases causing a beam widening. Moreover, the effect of matrix moves the peak of maximum intensity towards the greatest sampler torch distances. Since simultaneously with the ambipolar diffusion, the charge effect and the displacement of atomion equilibrium cause signal extinction along the central axis of plasma, the broadening of the signal is visible only far from this area, where the element of matrix has a weaker concentration.

Thus, the description of possible non-spectroscopic interferences allows to define parameters highlighting the origin of the interference and controllable by the experimenter. These parameters are the nebulizer flow, the addition of a transport modifier, observation position in the plasma, mass and electronegativity of elements.

The most studied matrices are $\mathrm{Na}, \mathrm{K}$ and $\mathrm{Ca}$ not only for historical reasons, since they were largely known in the techniques with flame, but also because they are most frequent in the environment (sea water, river or biological fluids) [6]. To be able to bring closer our results those of the literature and to validate our methodology, our study will also relate to $\mathrm{Na}, \mathrm{K}$ and $\mathrm{Ca}$. Since samples analyzed by ICP-MS in our laboratory being ores, slags, basalts, slags of refining, matrix mainly consist of $\mathrm{Si}$ and Mg, we decided to study alakali metals, rare earth metals and Si.

\section{Experimental}

\subsection{Instrumentation}

The icp-mass spectrometer used in this study is a quadrupole Thermo fisher Scientific X7 equipped with a Meinhard concentric nebulizer. The operation conditions are shown in Table 1. The parameters were chosen in such a way as to produce maximum analyte ion count rate thanks a mass calibration over the whole mass range performed each day and to provide minimal values of the ratios $\mathrm{CeO}^{+} / \mathrm{Ce}^{+}$and $\mathrm{Ba}^{2+} / \mathrm{Ba}^{+}$. This optimization also established the optimum compromise axial Z (sample depth), X (horizontal) and radial Y (vertical) positions according to the nomenclature of Holliday et al. [22]. The axial position is a measured of the relative distance between the load coil and the sampling cone. All spatial profiling was carried out under the best compromise multi-elemental conditions. One requirement for representative sampling of the plasma is the absence of a secondary discharge. Then we use some sort of torch shield (metal plate inserted between the torch and the load coil) in an attempt to mitigate such a discharge.

\subsection{Reagents and Solutions}

All reagents were of analytical grade. All multielemental solutions were prepared by diluting certified stock multielemental solution (SPEX, suprapur, Jobin Yvon). The solution containing the analyte elements only is a 10 $\mu \mathrm{g} / \mathrm{L}$ Al, V, Cr, Mn, Ni, Co, Cu, Zn, Ga, As, Ba, La, Ce and $\mathrm{Pb}$ in $2 \% \mathrm{HNO}_{3}$. It was used for mass calibration and all analyte elements measures without concomitant. These elements were chosen because they described the wall mass range, they have different ionization potentials and oxidation formation abilities (Table 2). Height matrix containing $1000 \mathrm{mg} / \mathrm{L}$ alkaline salts, alkaline earth metal salts or inorganic acids were prepared each time by diluting with $2 \% \mathrm{HNO}_{3}: \mathrm{NaNO}_{3}, \mathrm{KNO}_{3}, \mathrm{CsNO}_{3}, \mathrm{Ca}\left(\mathrm{NO}_{3}\right)_{2}$, $\mathrm{Mg}\left(\mathrm{NO}_{3}\right)_{2}$, elementary $\mathrm{Si}, \mathrm{HCl}$ and $\mathrm{HI}$ (Table 3). These matrix were taken as representative of the different concomitants present in liquid samples.

Table 1. Experimental conditions.

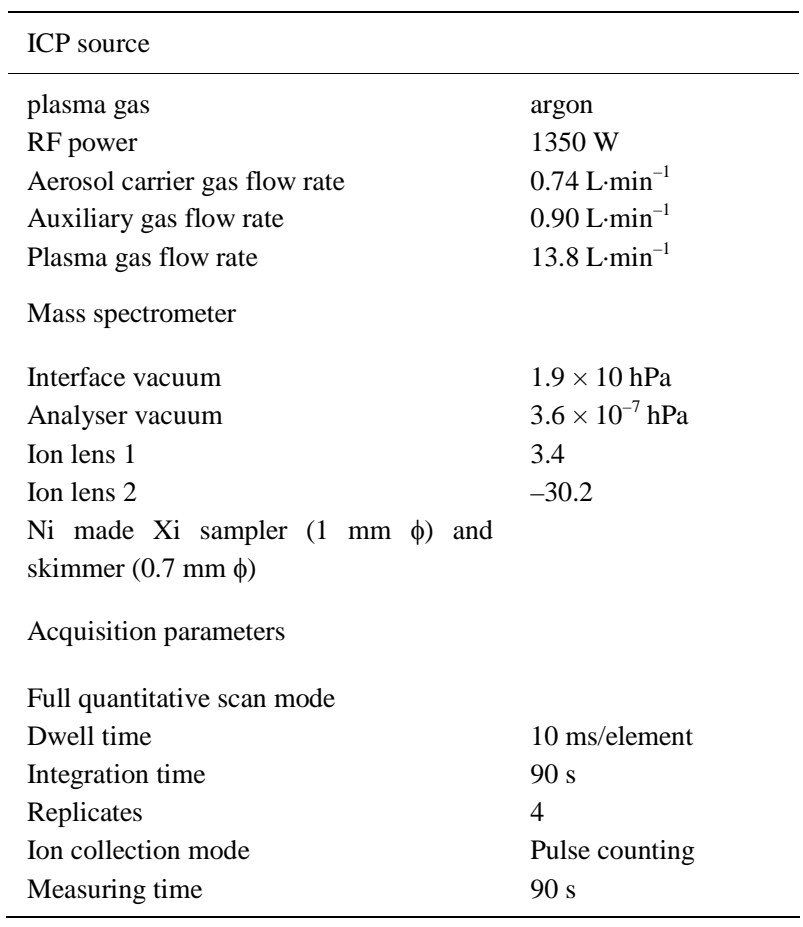


Table 2. Analytes properties.

\begin{tabular}{|c|c|c|c|c|c|c|}
\hline Element & $\begin{array}{l}\mathrm{m} / \mathrm{z} \\
\text { ratio }\end{array}$ & $\begin{array}{l}\text { Isotope aboundance } \\
(\%)^{\mathrm{d}}\end{array}$ & $\begin{array}{l}\text { First ionization } \\
\text { energy }(\mathrm{eV})^{\mathrm{a}}\end{array}$ & $\begin{array}{l}\text { Second ionization } \\
\text { energy }(\mathrm{eV})^{\mathrm{a}}\end{array}$ & $\begin{array}{l}\text { Ionization degree } \\
{(\%)^{\mathrm{b}}}^{-y^{2}}\end{array}$ & $\begin{array}{l}\text { Strengh of oxide bond } \\
\qquad(\mathrm{kJ} / \mathrm{mol})^{\mathrm{c}}\end{array}$ \\
\hline $\mathrm{Al}$ & 27 & 100 & 5.984 & 18.83 & 98 & 512.1 \\
\hline $\mathrm{V}$ & 51 & 99.76 & 6.74 & 14.65 & 99 & 626.8 \\
\hline $\mathrm{Cr}$ & 52 & 83.764 & 6.764 & 16.5 & 98 & 429.3 \\
\hline Mn & 55 & 100 & 7.435 & 15.64 & 95 & 402.9 \\
\hline $\mathrm{Ni}$ & 58 & 67.77 & 7.635 & 18.17 & 91 & 382 \\
\hline Co & 59 & 100 & 7.876 & 17.06 & 93 & 384,5 \\
\hline $\mathrm{Cu}$ & 63 & 69.17 & 7.726 & 20.29 & & \\
\hline $\mathrm{Zn}$ & 64 & 48.6 & 9.394 & 17.96 & & \\
\hline As & 75 & 100 & 9.814 & 18.63 & 52 & 481 \\
\hline In & 115 & 95.7 & 5.786 & 18.87 & & \\
\hline $\mathrm{Ba}$ & 138 & 71.7 & & & & 552 \\
\hline $\mathrm{La}$ & 139 & 99.911 & 5.577 & 11.06 & $90(10)^{\mathrm{e}}$ & 799 \\
\hline $\mathrm{Ce}$ & 140 & 88.48 & 5.466 & 10.85 & $96(2)^{\mathrm{e}}$ & 797 \\
\hline $\mathrm{Pb}$ & 208 & 52.4 & 7.415 & 15.03 & $97(0.01)^{\mathrm{e}}$ & 382 \\
\hline $\mathrm{Pb}^{2+}$ & 104 & & & & & \\
\hline $\mathrm{Ce}^{2+}$ & 70 & & & & & \\
\hline $\mathrm{Ba}^{2+}$ & 69 & & & & & \\
\hline $\mathrm{VO}^{+}$ & 145 & & & & & \\
\hline $\mathrm{BaO}^{+}$ & 154 & & & & & \\
\hline
\end{tabular}

a [24]; ${ }^{b}[21] ;{ }^{c}[25]$. It deals with the bond strength of neutral molecules; ${ }^{\mathrm{d}}[26]$; ${ }^{\mathrm{e}}$ Second ionization degree.

Table 3. Matrix elements properties.

\begin{tabular}{cccccc}
\hline Element & Atomic mass & $\begin{array}{c}\text { First ionization energy } \\
(\mathrm{eV})^{\mathrm{a}}\end{array}$ & $\begin{array}{c}\text { Second ionization } \\
\text { energy }(\mathrm{eV})^{\mathrm{a}}\end{array}$ & $\begin{array}{c}\text { Ionization degree } \\
(\%)^{\mathrm{b}}\end{array}$ & $\begin{array}{c}\text { Strengh of oxide bond } \\
(\mathrm{kJ} / \mathrm{mol})^{\mathrm{c}}\end{array}$ \\
\hline $\mathrm{Na}$ & 22.9898 & 5.14 & 47.28 & 100 & 256.1 \\
$\mathrm{Mg}$ & 24.3050 & 7.64 & 15.03 & & 277.8 \\
$\mathrm{Si}$ & 28.0855 & 8.15 & 16.34 & 100 & 295.8 \\
$\mathrm{~K}$ & 39.0983 & 4.34 & 31.62 & & \\
$\mathrm{Ca}$ & 40.078 & 6,11 & 11.87 & 100 & 25.08 \\
$\mathrm{Cs}$ & 132.9054 & 3.89 & & & \\
\hline
\end{tabular}

${ }^{\mathrm{a}}[24] ;{ }^{\mathrm{b}}[21]$; ${ }^{\mathrm{c}}[25]$; It deals with the bond strength of neutral molecules.

\subsection{Procedure}

The following procedure is used in order to determine the effect of concomitant elements on analyte ion count rates. The same set of four experiments was performed for each of the concomitant elements. Each experiment consisted of four repetitions: first with a blank consisting only of $2 \% \mathrm{HNO}_{3}$ t; the second with the multielement solution; the third with a blank $2 \% \mathrm{HNO}_{3}$ solution containing the concomitant element; and the fourth with a multielement solution containing the concomitant element. The multielement solution not containing the concomitant element was repeated for every experiment in order to account for day-to-day variability in sensitivity. Five replicates were taken at each measurement incre- ment before advancing to the next point of interest. Thanks the computer-controlled translation stage of the instrument four experiments were performed: (1) axial profiles by moving the torch away from the sampler along the central axis of the plasma to get a measurement of the signal intensity at a variety of sampling depths; (2) radial signal profile of the ion distribution across the central channel of the plasma by moving the torch across the sampler at each of three fixed sampling depths $(\mathrm{Z}=$ optimum, $Z=$ optimum +50 and $Z=$ optimum +100$)$, respectively.

\subsection{Matrix Effect Measures}

ME may be defined as matrix-induced signal variations, 
either enhancement or suppression of the analyte signal. In this paper, like some other authors [23-26], we use the suppression degree defined as the variation of net intensities using the following formula:

$$
\text { Suppression degree }=\frac{I(A)_{m}-I(\text { blank })_{m}}{I(A)-I(\text { blank })}-1
$$

In this relation (1), we compare the analyte signals intensities $\mathrm{I}(\mathrm{A})_{\mathrm{m}}$ with and without matrix I(A), after correction of the blank was involved since we subtracted blank with I(blank $)_{m}$ and without matrix I(blank). We can tell there is extinction when the suppression degree is lower than 0 , and there is enhancement if it is higher than 0 .

\section{Results and Discussion}

Error bars are not included in the figures below in order to avoid confusion in the already congested plots. An average of five measurements was taken for each run and triplicate runs were used to determine the relative standard deviation (R.S.D.). Here, a measurement consists of single integration of the value, which was repeated five times for each run. The R.S.D. of the runs, obtained from triplicates taken on a given day, was less than $5 \%$. The only exceptions to this situation are the plots for barium ion emission and number density. These plots and the reasons for the discrepancy will be discussed in detail later.

\subsection{Comparison of Signal of Analytes from Mul- ti-Elemental Solutions in Different Con- comitant Elements}

First, to highlight the relation between the signal extinction of an analyte and the masse of the concomitant element, we studied the influence of different masse elements but in the same chemical family. We compared the effects of the alkaline ( $\mathrm{Na}, \mathrm{K}, \mathrm{Cs}$ ) on the one hand and alkaline earth metal (Mg, Ca) and Si on the other hand. From this way, there is almost no influence of energy of ionization and only the space charge effect will be highlighted.

The analyte signal with and without the element of matrix will be recorded for several sampling depths. The evolution of the degree of suppression according to sampling depth for each matrix shows the same trend for several analytes. Thus, we obtained practically the same curves for $\mathrm{V}^{+}, \mathrm{Cr}^{+}, \mathrm{Mn}^{+}, \mathrm{Ni}^{+}, \mathrm{Cu}^{+}, \mathrm{Zn}^{+}$and $\mathrm{Co}^{+}$for the one hand, and the nearly same curves $\mathrm{Ce}^{+}, \mathrm{Ba}^{+}$and $\mathrm{La}^{+}$ on the other hand, like illustrated Figures 1and 2. The addition of an EIE contributes a large number of electrons low in the plasma (where the electron number density is low) and results in a shift in the analyte ion-atom equilibrium taking place. Cs releases more electrons than
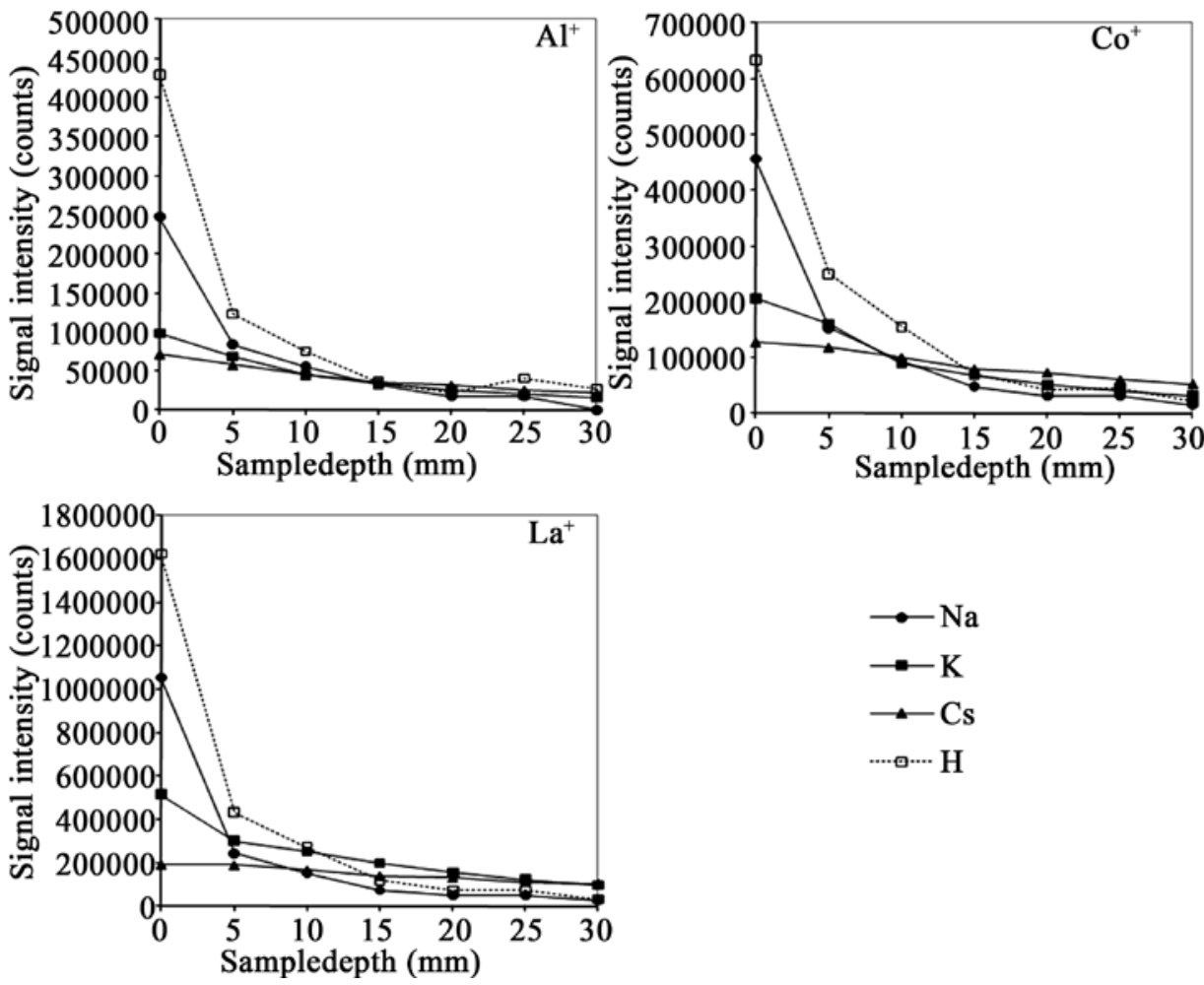

Figure 1. Comparison of signal intensities for $\mathrm{Al}^{+}, \mathrm{Co}^{+}$and $\mathrm{La}^{+}$in several alkaline matrix. 

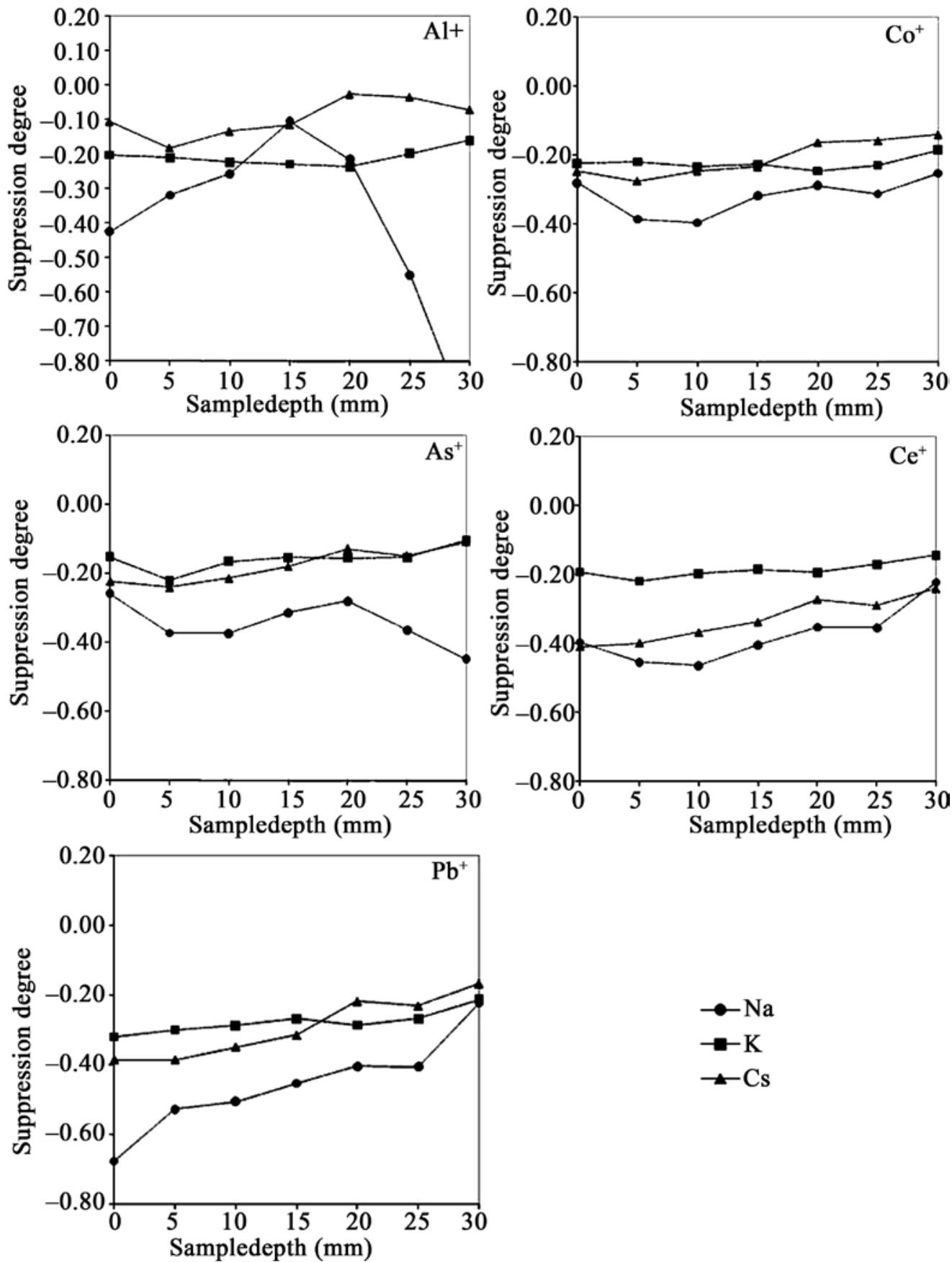

Figure 2. Suppression degree from multi-elemental solutions in different concomitant elements.

$\mathrm{Na}$, hence reducing the ion population in this region. It explains that the signal intensities for Cs is always lower than for Na (Figure 1). Cs suppresses more than Na because it is heavier and has a lower ionization potential, thus exacerbating space charge effects. For one mass, for example $\mathrm{Al}$ or $\mathrm{Pb}$, the suppression degree in $\mathrm{Na}$ is lower when the sample depth is 0 because the electron density is more important where the ionization of Na takes place. When the torch is moved away from the sampler, the suppression degree increases, the electron density due to the EIE is lower because of the diffusion of the electron. And for very high sample depth (or for lighter analytes like $\mathrm{Al}$ ), the suppression degree decreases but it is because of there are very few analyte ions in this place of the plasma.

For the weak masses analytes $(\mathrm{m} / \mathrm{z} \leq 50)$, the suppre ssion degree follows the first ionization energy of matrix element (Table 1) while there is an inversion between $\mathrm{K}$ 
and Cs when analyte mass increases (Figure 2). For $\mathrm{m} / \mathrm{z}$ $\geq 50$, the influence of the ionization energy is combined with the influence of the mass of the EIE, the space charge effect takes place and is all the more stronger since the analyte is heavier. We can note that it is there is no influence of first ionization energy of the analyte since $\mathrm{Al}$, La and Ce (Table 2) have almost the same one but don't present the same suppression degree. As we obtained the same curves for analyte in the same mass range, we can think the suppression degree for a given concomitant element only depends on the analyte mass. For the analytes studied, it was found that the greater the atomic mass of the concomitant element, the greater was
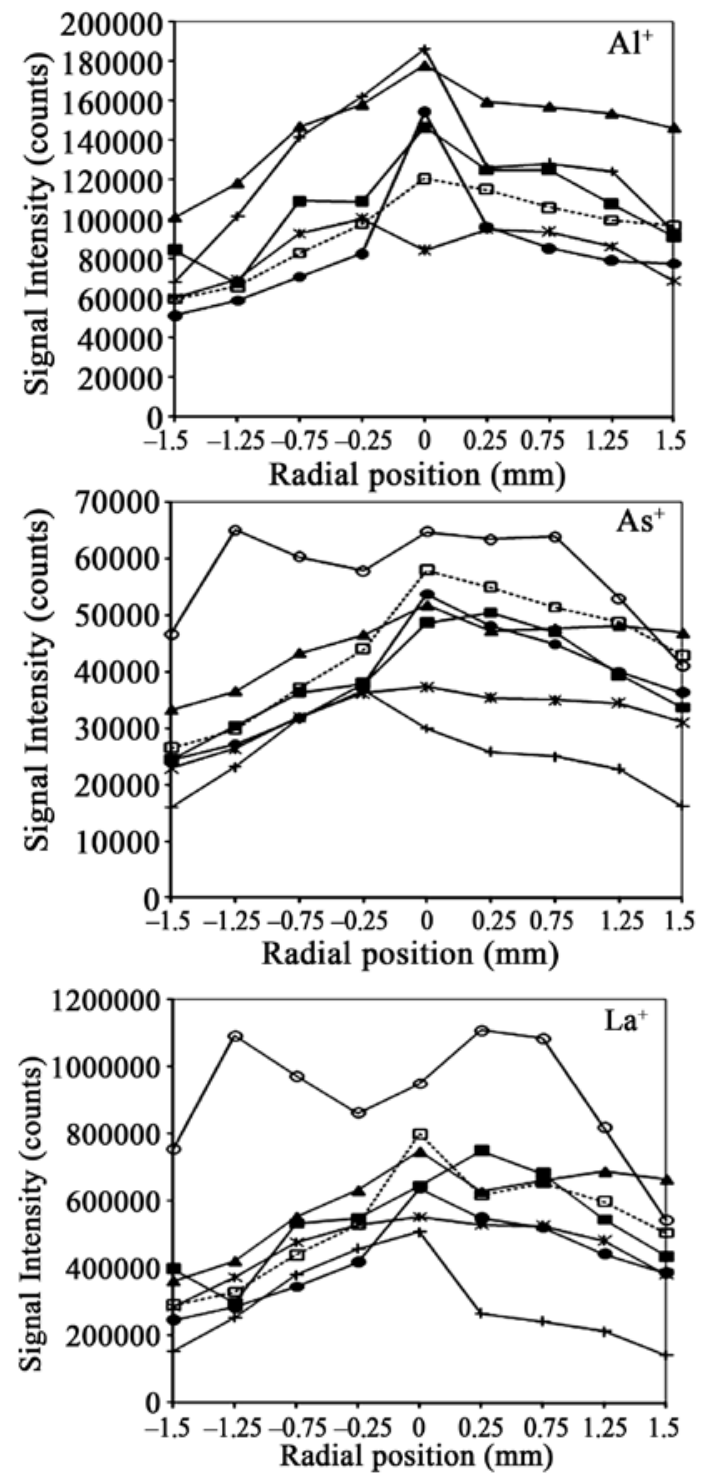

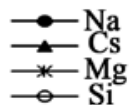

the analyte ion count rate suppression. These results allowed us to conclude that in the alkali metals and in the rare earth metal series, we observed the influence of the space charge effect.

Figures 3 and 4 illustrate the radial distribution of elements within the plasma. From these data it can be seen that despite large variations in absolute signal intensity, the radial distribution is nearly identical for all analytes. The graphs of the radial distribution of the ions in the presence EIE reveal that most of the elements, like As, In, La are slightly suppressed within $1 \mathrm{~mm}$ of the central axis a part with $\mathrm{Si}$.
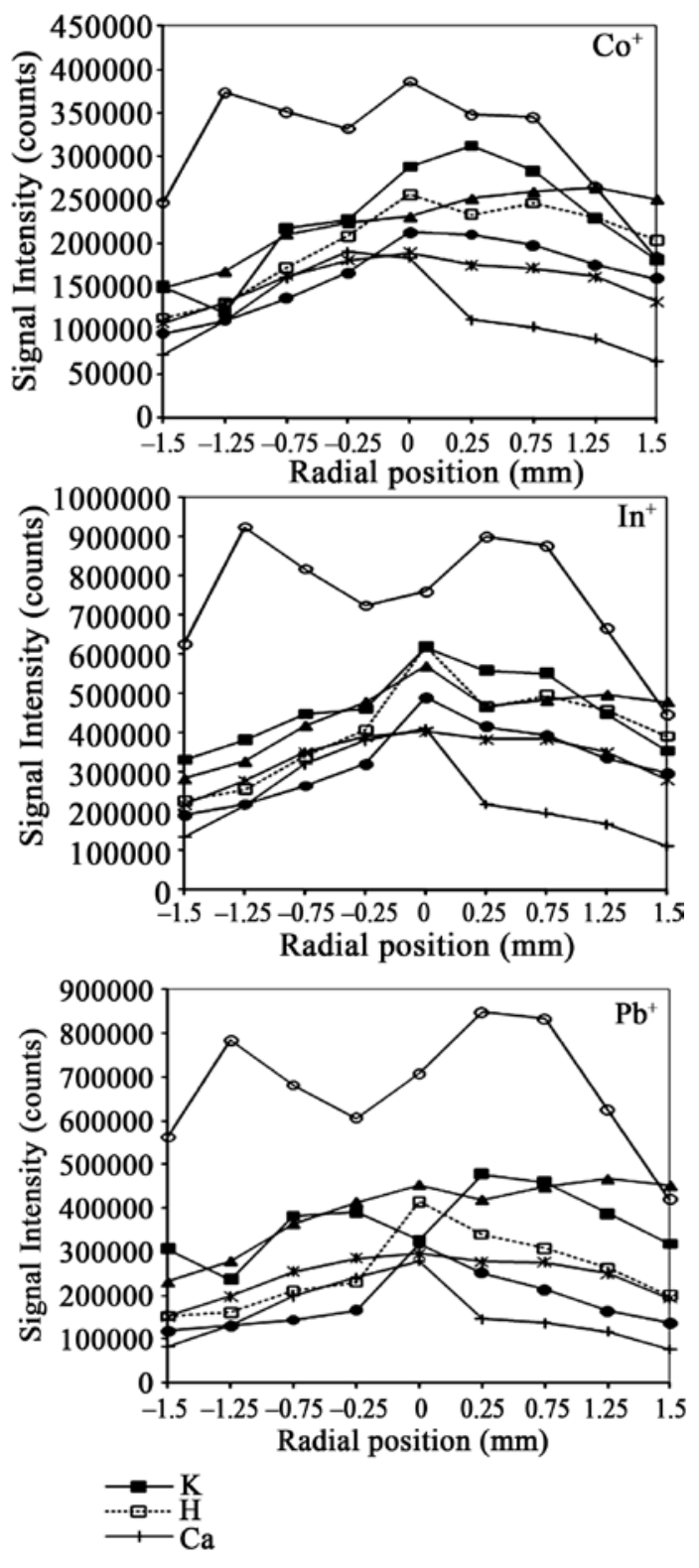

Figure 3. Comparison of signal intensity for several elements in different matrix. 

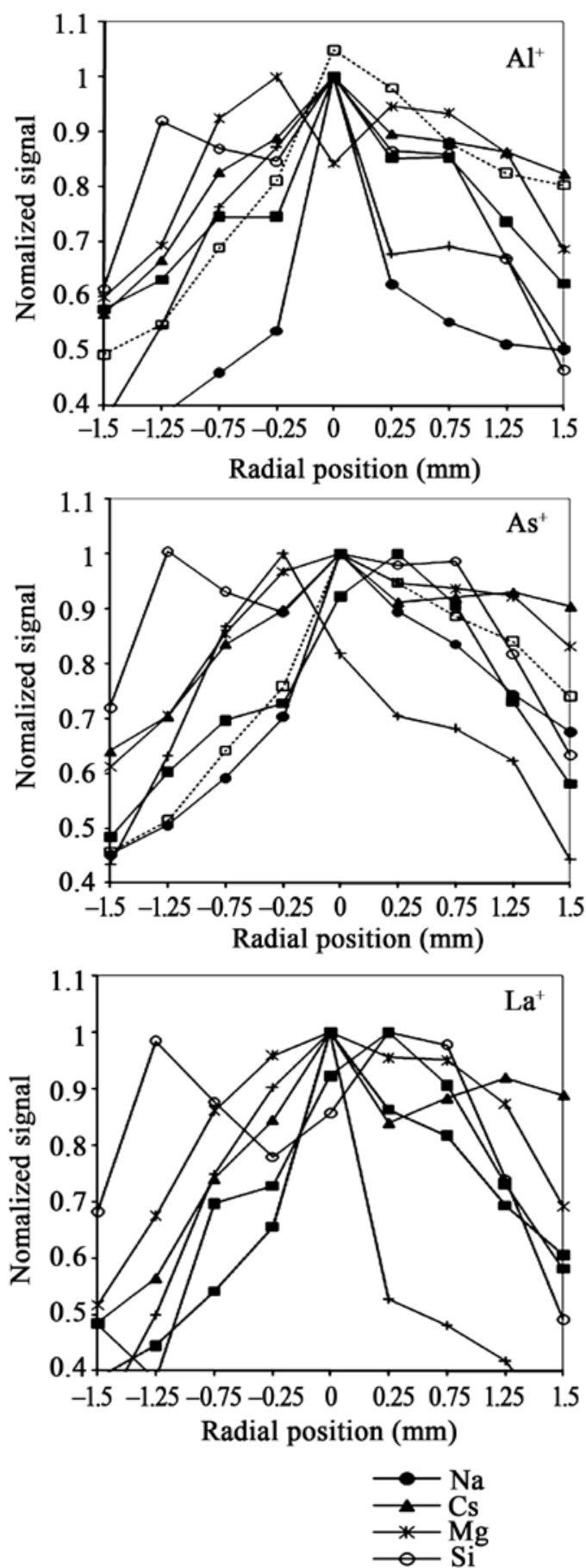
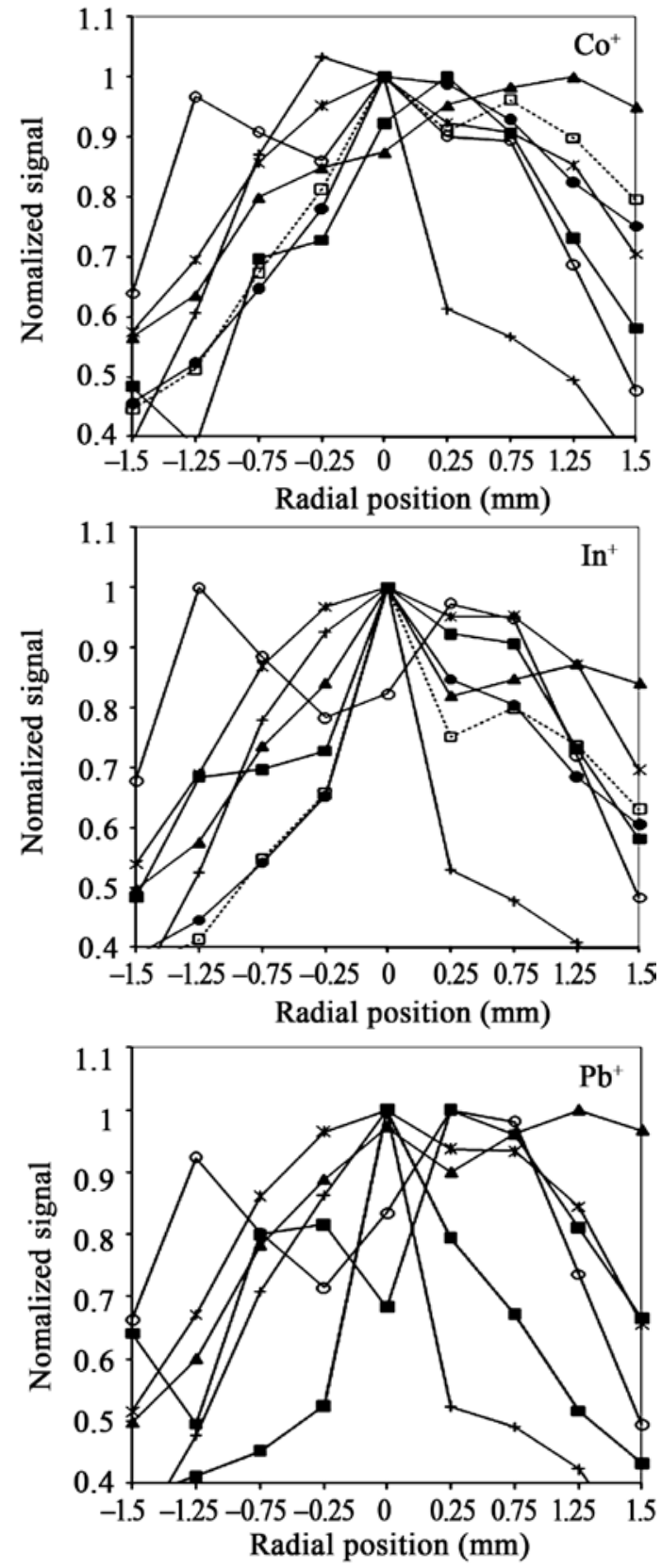

$-\mathrm{K}$
$\cdots \mathrm{H}$
$+\cdots \mathrm{Ca}$

Figure 4. Comparison of normalized signal for several elements in different matrix.

In some cases, the signals appear to be slightly enhanced outside of this region (also referred to as broadening). This broadening is more apparent in the graph illustrating the normalized signal (Figure 4).

The heaviest matrix element examined was cesium. Its mass is considerably heavier than the others EIE examined, so we expected the suppression in its presence was much greater than that due to the others EIE examined. Furthermore Cs also has the lowest first ionization energy of any of the matrix elements examined. Finally these two combined factors don't make the largest matrix effect, then the matrix effect of Cs origins in many phenomenon.

To highlight the relation between the removal of the 
analyte signal and the first ionization energy of the element of matrix, we studied the influence of elements of close masses but different chemical families. So, we compared effects of the couples (K, Ca) and ( $\mathrm{Na}, \mathrm{Mg})$. In this time there is no influence of the mass of the matrix element, and it is the displacement of ion-atom equilibrium which will be highlighted.

$\mathrm{Ca}$ and $\mathrm{K}$ have very close masses; only theirs ionization energies are different. Apart from Al, Ca involves a greater extinction than $\mathrm{K}$. In the same way $\mathrm{Mg}$ leads to a larger broadening of signals than Na.

The "bell" shaped ion distribution seen for the analytes is totally suppressed in the presence of Si matrix. The signal profiles are almost flat with a slight local minimum along the central axis. The minimum is more marked when analyte is heavier. Examining the normalized signal intensity for all the analytes (Figure 4) reveals that despite the overall flattened appearance of the analyte signal, there is still some variation in signal intensity with radial position. The same results are obtained for $\mathrm{Pb}$ in $\mathrm{K}$ or Cs matrix.

Such broadening of signals indicates that ions are diffusing more quickly than normal in the plasma which would be a logical consequence of increasing the density of electrons in the plasma, it is ambipolar diffusion.

\subsection{Comparison of Signal of Dications and Oxides from Multi-Elemental Solutions in Different Concomitant Elements}

In a second time we focused on the doubly charged ions. Signal and suppression degree have the same variations for dications than for monocations (Figure 5). We chose represent $\mathrm{Ba}^{2+}$, but the same trends were observed for $\mathrm{Ce}^{2+}$ and $\mathrm{Pb}^{2+}$. Examining radial profiles of the dications (Figure 6) reveals that they appear to be broadened slightly more than the other analytes in the presence of the same matrix element at the same sample depth. This broadening increases with decreasing matrix element ionization energy. There is no dependence on matrix or analyte element rnasses. Figure 7 shows the dications are formed at great sample depth, where the plasma is hottest and the electron density is lowest. These observations are in agreement with a displacement of ion - atom equilibrium of the doubly ionized elements.

To study the matrix effect on oxides formation and where these processes take place, we profile analyte oxides (Figures $\mathbf{7}$ and $\mathbf{8}$ ). Three of the analytes (V, La, $\mathrm{Ba}$ and $\mathrm{Ce}$ ) form oxides in the plasma, and have distinctly different behavior than the remainder of the elements examined. Note that in both of the above tables the oxide bond strengths are for diatomic molecules, not ions. The signal intensity of $\mathrm{CeO}^{+}$is not representative of the true distribution within the plasma because the number of counts is not high enough. The distribution of the ions is in agreement with what was expected (Figure 7). The oxides are formed at low sample depth where the plasma is somewhat cooler. As they are carried to greater depths, they are then dissociated into their respective analytes which results in the analyte signals intensity increasing with sampling distance. The radial profiles of $\mathrm{BaO}^{+}$are usually very similar to those of $\mathrm{Ba}^{+}$. Oxides' formation seems mainly depends on energy demand in the plasma (Figure 8).
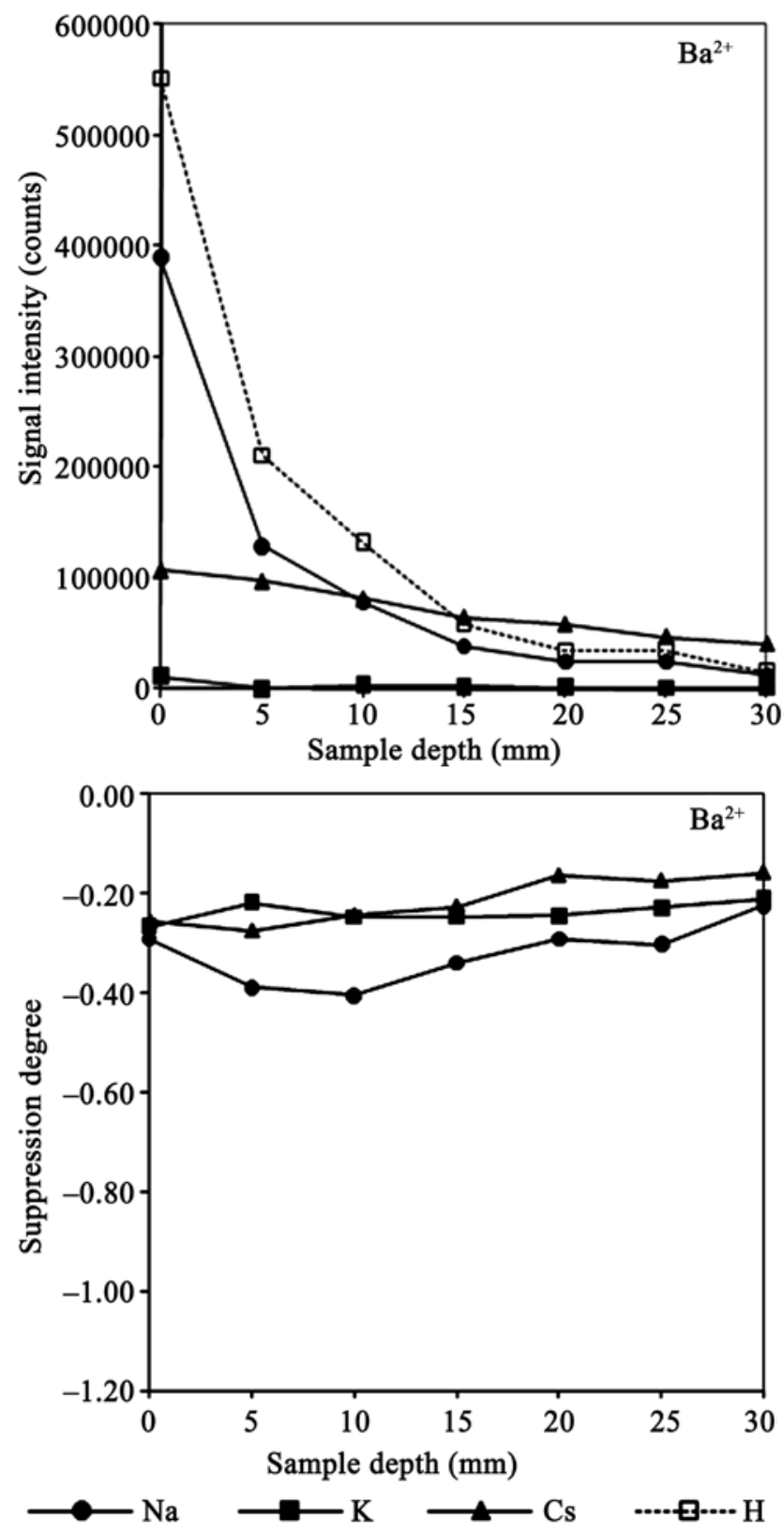

Figure 5. Variations of signal intensities for $\mathrm{Ba}^{2+}$ in several matrix with sample depth. 

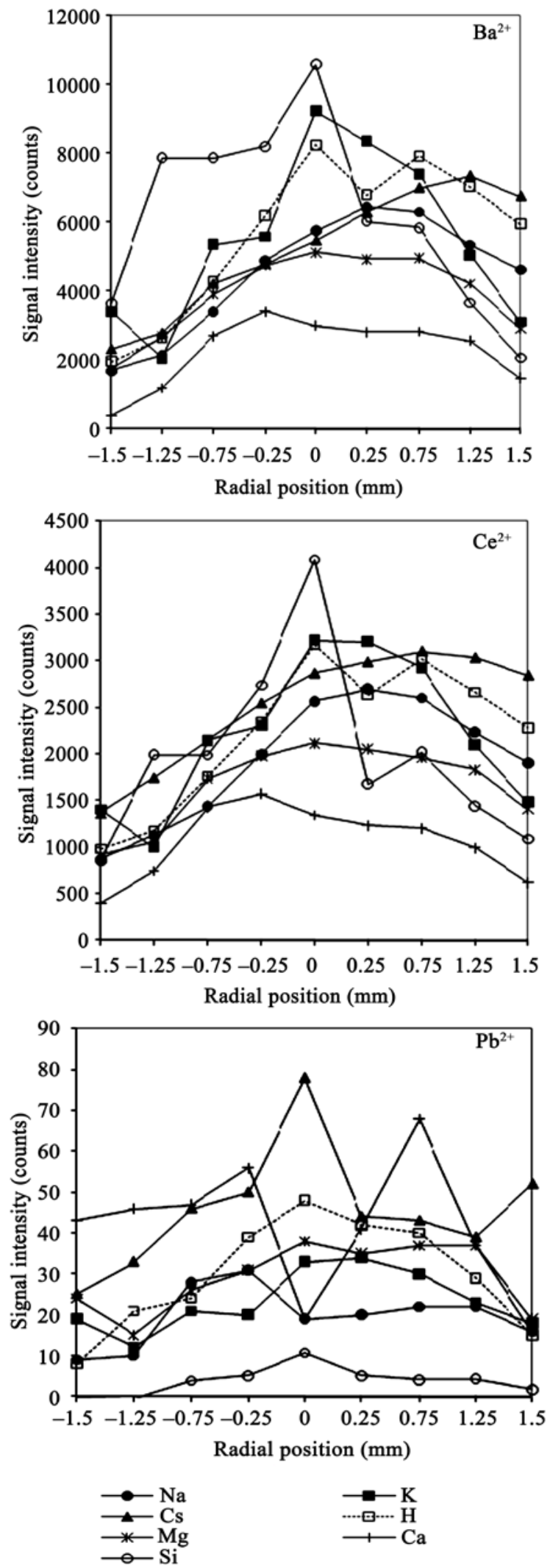

Figure 6. Variations of signal intensities for $\mathrm{Ba}^{2+}, \mathrm{Ce}^{2+}$ and $\mathrm{Pb}^{2+}$ in several matrix with radial position.
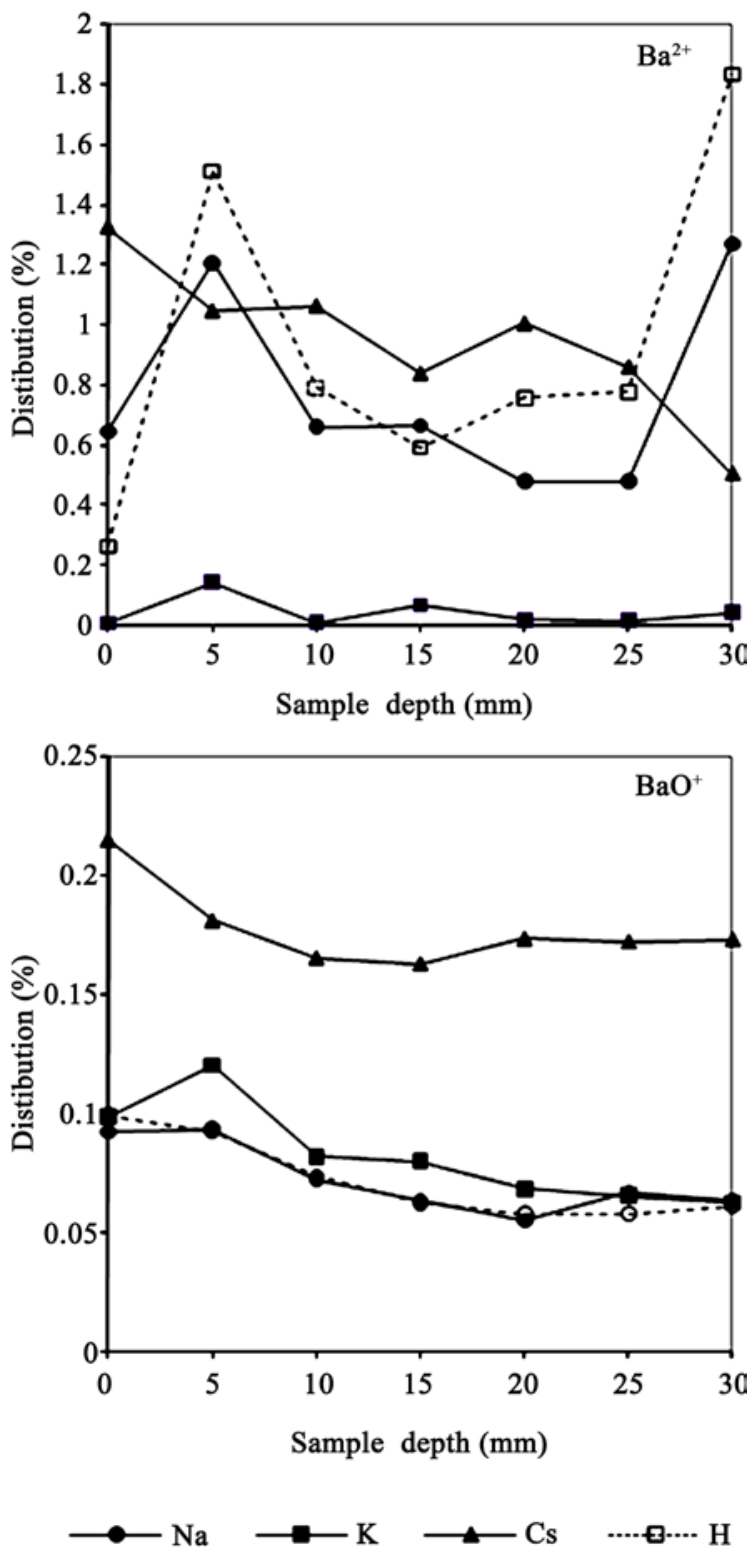

Figure 7. Comparison of signal intensities for $\mathrm{Ba}^{2+}$ and $\mathrm{BaO}^{+}$in several alkaline matrix

\section{Conclusions}

Comparing the data with and without the matrix elements allows us to conclude that the mass of the matrix element is definitely an important factor in matrix effects, and to better understand the nature of the matrix effects that occur in the ICP. A dependence upon both the mass of the matrix element and the mass of the analyte was observed. The suppression seems increased with increasing matrix element mass and decreased with increasing analyte mass. The effect of the mass of the matrix element was the more significant of the two factors. Mainly space-charge effects were found to be significant 

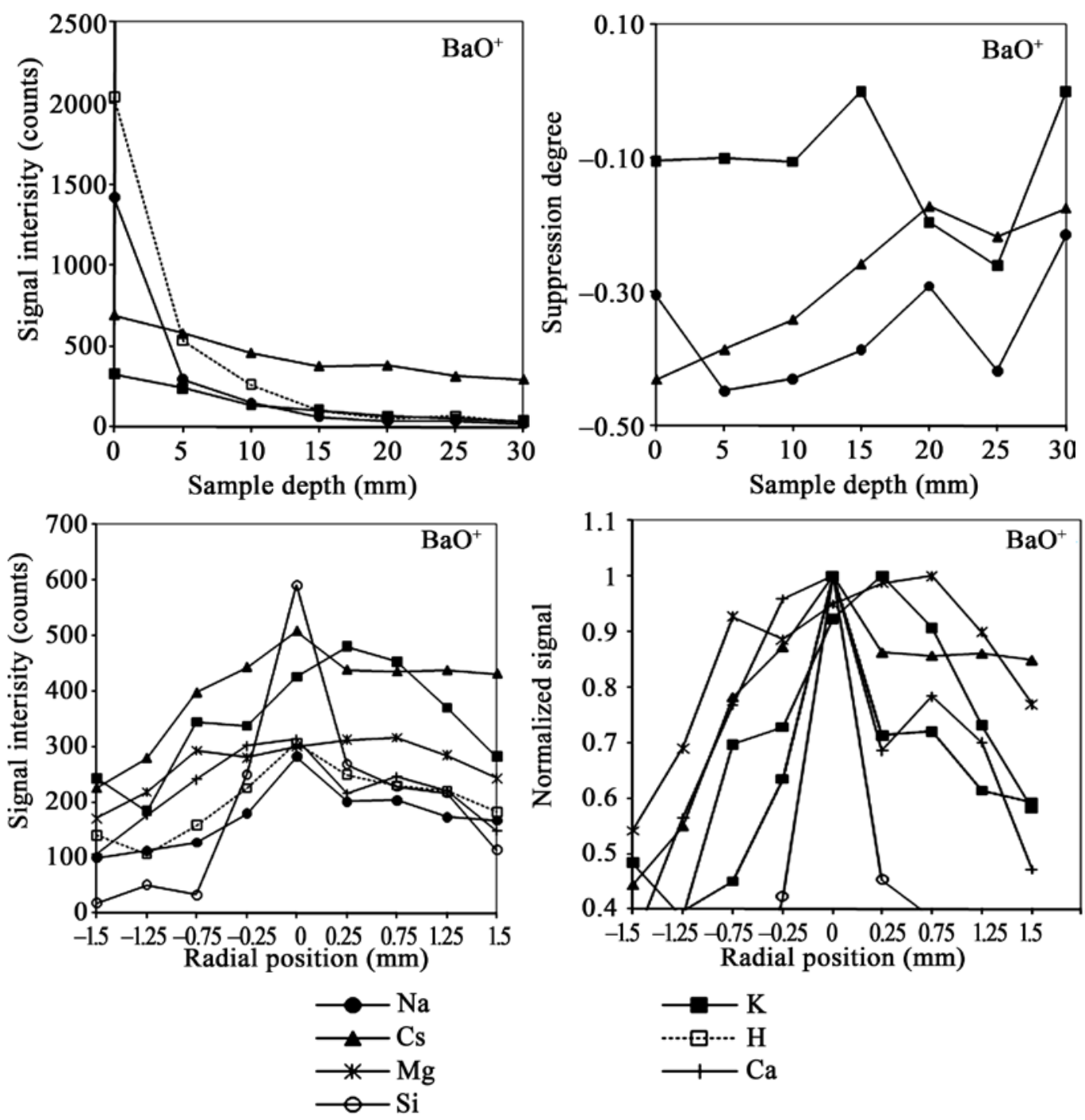

Figure 8. Comparison of $\mathrm{BaO}^{+}$signal intensities in several matrix.

for matrix elements of much lower mass. As these effects take place behind the cones, in the interface, we have to modify voltage of extraction lens to reduce matrix effects in this case. For heavier matrix elements, it seems not only space charge effect but also diffusion played an active part in the signal modification. Once more, we have to correct voltage of extraction lens but it is also necessary to move the torch in the radial direction to optimize signal. Finally, some evidence was found for a shift in ion-atom equilibrium for dications and for energy demand regarding oxides. In addition, all measurements were performed systematically on the same plasma so a better description of ICP behavior can be developed.

\section{References}

[1] D. D. Nygaard, "Plasma Emission Determination of
Trace Heavy Metals in Salt Water Matrics," Analytical Chemistry, Vol. 51, No. 7, 1979, pp. 881-884. doi:10.1021/ac50043a024

[2] J. W. Olesik, "Elemental Analysis Using ICP-OES and ICP/MS,” Analytical Chemistry, Vol. 63, No. 1, 1991, pp. 12A-21A. doi:10.1021/ac00001a001

[3] A. C. Lazar and P. B. Farnsworth, "Matrix Effect Studies in the Inductively Coupled Plasma with Monodisperse Droplets. Part I: The Influence of Matrix on the Vertical Analyte Emission Profile," Applied Spectroscopy, Vol. 53, No. 4, 1999, pp. 457-464. doi:10.1366/0003702991946749

[4] V. Karanassios and G. Horlick, "Elimination of Some Spectral Interferences and Matrix Effects in Inductively Coupled Plasma-Mass Spectrometry Using Direct Sample Insertion Techniques," Spectrochimica Acta Part B: Atomic Spectroscopy, Vol. 44, No. 12, 1989, pp. 13871396. doi:10.1016/0584-8547(89)80131-4 
[5] J. L. Venzie and R. K. Marcus, "Effects of Easily Ionisable Elements on the Liquid Sampling-Atmospheric Pressure Glow Discharge," Spectrochimica Acta Part B, Vol. 61, No. 6, 2006, pp. 715-721. doi:10.1016/j.sab.2006.02.005

[6] G. C. -Y. Chan and G. M. Hieftje, "Investigation of Plasma-Related Matrix Effects in Inductively Coupled Plasma-Atomic Emission Spectrometry Caused by Matrices with Low Second Ionization Potentials- Identification of the Secondary Factor," Spectrochimica Acta Part B, Vol. 61, No. 6, 2006, pp. 642-659. doi:10.1016/j.sab.2005.09.007

[7] G. Gamez, S. A. Lehn, M. Huang and G. M. Hieftje, "Effect of Mass Spectrometric Sampling Interface on the Fundamental Parameters of an Inductively Coupled Plasma as a Function of Its Operating Conditions Part I. Applied r.f. Power and Vacuum," Spectrochimica Acta Part B, Vol. 62, No. 4, 2007, pp. 357-369. doi:10.1016/j.sab.2007.03.015

[8] G. Gamez, S. A. Lehn, M. Huang and G. M. Hieftje, "Effect of Mass Spectrometric Sampling Interface on the Fundamental Parameters of an Inductively Coupled Plasma as a Function of Its Operating Conditions Part II. Central-Gas Flow Rate and Sampling Depth,” Spectrochimica Acta Part B, Vol. 62, No. 4, 2007, pp. 370-377. doi:10.1016/j.sab.2007.03.016

[9] S. A. Lehn, K. A. Warner, M. Huang and G. Hieftje, "Effect of Sample Matrix on the Fundamental Properties of the Inductively Coupled Plasma," Spectrochimica Acta Part B, Vol. 58, No. 10, 2003, pp. 1786-1806. doi:10.1016/S0584-8547(03)00159-9

[10] D. Lariviere, V. F. Taylor, R. D. Evans and R. J. Cornett, "Radionuclide Determination in Environmental Samples by Inductively Coupled Plasma Mass Spectrometry," Spectrochimica Acta Part B, Vol. 61, No. 8, 2006, pp. 877-904. doi:10.1016/j.sab.2006.07.004

[11] A. M. Desaulty, C. Mariet, P. Dillmann, J. L. Joron and P. Fluzin, "A Provenance Study of IroN Archaeological Artefacts by ICP-MS Multi-Elemental Analysis,” Spectrochimica Acta Part B, Vol. 63, No. 11, 2008, pp. 12531262. doi:10.1016/j.sab.2008.08.017

[12] M. He, B. Hu, Y. Zeng and Z. Jiang, "ICP-MS Direct Determination of Trace Amounts of Rare Earth Impurities in Various Rare Earth Oxides with Only One Standard Series," Alloys and Compounds, Vol. 390, No. 1-2, 2005, pp. 168-174. doi:10.1016/j.jallcom.2004.06.107

[13] S. Kozono and H. Haraguchi, "Determination of Ultratrace Impurity Elements in High Purity Niobium Materials by on-Line Matrix Separation and Direct Injection/Inductively Coupled Plasma Mass Spectrometry,” Talanta, Vol. 72, No. 5, 2007, pp. 1791-1799. doi:10.1016/j.talanta.2007.02.021

[14] T. Duan, X. Song, P. Guo, H. Li, L. Pan, H. Chena and J. $\mathrm{Xu}$, "Elimination of Matrix Effect and Spectroscopic Interference by Two Compactly Combined Separations in the Determination of Cd in Geological Samples with High Mo, Zr or Sn Contents by ICP-MS,” Journal of Analytical and Atomic Spectrometry, Vol. 22, No. 4, 2007, pp. 403-406. doi:10.1039/b610685d

[15] B. U. Peschel, W. Herdering and J. A. C. Broekaert, “A Radiotracer Study on the Volatilization and Transport Effects of Thermochemical Reagents Used in the Analysis of Alumina Powders by Slurry Electrothermal Vaporization Inductively Coupled Plasma Mass Spectrometry,” Spectrochimica Acta Part B, Vol. 62, No. 2, 2007, pp. 109-115. doi:10.1016/j.sab.2007.01.006

[16] J. Mora, L. Gras, E. H. van Veen and M. T. C. de Loos-Vollebregt, "Electrothermal Vaporization of Mineral Acid Solutions in Inductively Coupled Plasma Mass Spectrometry: Comparison with Sample Nebulization," Spectrochimica Acta Part B, Vol. 54, No. 6, 1999, pp. 959-974. doi:10.1016/S0584-8547(99)00029-4

[17] T. Ka'ntor, S. Maestre and M. T. C. D. Loos-Vollebregt, "Studies on Transport Phenomena in Electrothermal Vaporization Sample Introduction Applied to Inductively Coupled Plasma for Optical Emission and Mass Spectrometry," Spectrochimica Acta Part B, Vol. 60, No. 9-10, 2005, pp. 1323-1333. doi:10.1016/j.sab.2005.06.011

[18] D. C. Gregoire, "The Effect of Easily Ionisable Concomitant Elements on Non-Spectroscopic Interferences in Inductively Coupled Plasma Mass Spectrometry," Spectrochimica Acta Part B, Vol. 42, No. 6, 1987, pp. 895907. doi:10.1016/0584-8547(87)80100-3

[19] M. M. Fraser and D. Beauchemin, "Effect of Concomitant Elements on the Distribution of Ions in Inductively Coupled Plasma Mass Spectrometry. Part 1 Elemental Ions,” Spectrochimica Acta Part B, Vol. 55, No. 11, 2000, pp. 1705-1731. doi:10.1016/S0584-8547(00)00273-1

[20] J. A. Olivares and R. S. Houk, "Ion Sampling for Inductively Coupled Plasma Mass Spectrometry,” Analytical Chemistry, Vol. 57, No. 13, 1985, pp. 2674-2679. doi:10.1021/ac00290a054

[21] X. Chen and R. S. Houk, "Spatially Resolved Measurements of Ion Density behind the Skimmer of an Inductively Coupled Plasma Mass Spectrometer," Spectrochimica Acta Part B, Vol. 51, No. 1, 1996, pp. 41-54. doi:10.1016/0584-8547(95)01387-3

[22] A. E. Holliday and D. Beauchemin, "Spatial Profiling of Analyte Signal Intensities in Inductively Coupled Plasma Mass Spectrometry," Spectrochimica Acta Part B, Vol. 59, No. 3, 2004, pp. 291-311. doi:10.1016/j.sab.2003.12.018

[23] M. M. Fraser and D. Beauchemin, "Effect of Concomitant Elements on the Distribution of Ions in Inductively Coupled Plasma Mass Spectrometry. Part 2 Polyatomic Ions," Spectrochimica Acta Part B, Vol. 56, No. 12, 2001, pp. 2479-2495. doi:10.1016/S0584-8547(01)00346-9

[24] R. S. Houk, "Mass Spectrometry of Inductively Coupled Plasmas,” Analytical Chemistry, Vol. 58, No. 1, 1986, pp. 97A-105A. doi:10.1021/ac00292a003

[25] H. Ying, M. Antler, J. W. Tromp and E. D. Salin, "Sample Diagnosis Using Non-Analyte Signals for Inductively Coupled Mass Spectrometry," Spectrochimica Acta Part B, Vol. 57, No. 2, 2002, pp. 277-290. doi:10.1016/S0584-8547(01)00382-2 
[26] M. T. Larrea, B. Zaldivar, J. C. Farinas, L. G. Firgairad and M. Pomares, "Matrix Effect of Aluminium, Calcium and Magnesium in Axially Viewing Inductively Coupled Plasma Atomic Emission Spectrometry," Journal of Analytical Atomic Spectrometry, Vol. 23, 2008, pp. 145-151. doi:10.1039/b709359d

[27] D. F. Schriver, P. W. Atkins and C. H. Langford, "Inor- ganic Chemistry,” W. H. Freeman and Company, New York, 1990.

[28] D. R. Lide, "CRC Handbook of Chemistry and Physics," 90th Edition, CRC Press, Boca Raton, 2009.

[29] S. H. Tan and G. Horlick, "Background Spectroscopic Features in Inductively Coupled Plasma/Mass Spectrometry," Applied Spectroscopy, Vol. 40, 1986, pp. 445460. doi:10.1366/0003702864508944 